\title{
Sylvia: Zur Entstehung des wissenschaftlichen Namens der Grasmücke (Arist. Hist. an. 592b22)
}

\author{
Grigory Vorobyev \\ Institute for Linguistic Studies, Russian Academy of Sciences, \\ 9, Tuchkov per., St. Petersburg, 199053 Russian Federation; \\ Ludwig Boltzmann Institute for Neo-Latin Studies, \\ 11, Langer Weg, Innsbruck, 6020 Austria; g_voro01@uni-muenster.de
}

For citation: Grigory Vorobyev. Sylvia: Zur Entstehung des wissenschaftlichen Namens der Grasmücke (Arist. Hist. an. 592b22). Philologia Classica 2018, 13(2), 247-264. https://doi.org/10.21638/11701/ spbu20.2018.206

The article aims at demonstrating that the Latin bird name sylvia used by modern zoologists originates from the Greek ü $\lambda a c$, attested in several manuscripts of Aristotle's Historia animalium (592b22). Working on his Latin translation of this treatise in the 1450s-1470s, Theo-

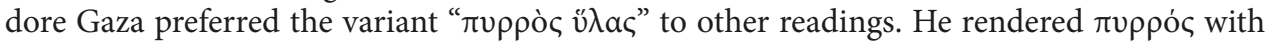
a neologism rubicilla and ü $\lambda \alpha \varsigma$ with the word silvia used only as a proper name before. As all the editions of the Greek text from the $1504 \mathrm{ed}$. princeps onwards take over Aldus Manutius' emendation $\pi u \rho \rho o v i \lambda a \varsigma$, the origin of silvia has not been ascertained until now, even though

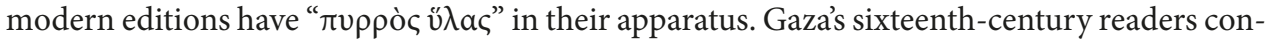
sidered silvia (later mostly spelled sylvia) a synonym of the nearby word rubecula, another neologism of his coinage, identified arbitrarily since the late fifteenth century with robin redbreast. That is why in 1769 Giovanni Antonio Scopoli used the word sylvia as the name for his newly introduced bird genus comprising robin redbreast. In 1800 Georges Cuvier suggested leaving only the typical warblers in that genus ("typical warblers" is a problematic name, since also whitethroats and other birds belong to the genus; the German Grasmücken is taxonomically more convenient), whereas other species, including robin redbreast, were moved to new genera. Thus, the word silvia coined as an equivalent for the Greek ü $\lambda$ ac (which was perhaps a scribal error) had been considered a name of robin redbreast for three centuries. Since 1800, spelled sylvia, it designates typical warblers.

Keywords: early modern zoological nomenclature, Latin neologisms, Aristotle's Historia animalium, warblers, robins, sylvia, silvia, pyrrhula, erithacus, rubecula.

(C) St. Petersburg State University, 2018 


\section{Die handschriftliche Überlieferung von Arist. Hist. an. $592 \mathrm{~b} 22$}

Die Grasmücken sind eine umfangreiche Vogelgattung aus der Familie der Grasmückenartigen, die ihrerseits zur Ordnung der Sperlingsvögel (Passeriformes) gehört. ${ }^{1}$ Der wissenschaftliche Name dieser Gattung, Sylvia (Scopoli, 1769), ${ }^{2}$ von dem auch der Familienname Sylviidae abgeleitet ist, ${ }^{3}$ hat in sich auf den ersten Blick nichts Besonderes. ${ }^{4}$ Wohl deswegen ist die Entstehung dieses Vogelnamens in der Forschungsliteratur bislang nicht untersucht worden. ${ }^{5}$ Als das Wort Sylvia 1769 von dem italienisch-österreichischen Naturforscher Giovanni Antonio Scopoli als Gattungsbezeichnung der Grasmücken eingeführt wurde, hatte es schon eine lange Geschichte. Sie beginnt mit einer offenbar korrupten Lesart in Aristoteles' Historia animalium.

Im Buch 8 zählt Aristoteles u. a. die Vögel auf, die $\sigma \kappa \omega \lambda \eta \kappa о \varphi \alpha ́ \gamma \alpha$ sind, d. h. sich von Würmern bzw. Insektenlarven ernähren:

${ }^{1}$ Die Arbeit am vorliegenden Aufsatz wurde durch meinen von Mai bis September 2018 stattgefundenen Aufenthalt als research fellow im Ludwig Boltzmann Institut für neulateinische Studien (Innsbruck) ermöglicht. Ich danke ganz herzlich Martin Korenjak, Dominik Berrens und anderen Mitarbeitern des Instituts sowie des ERC-Projekts „Noscemus“, mit denen ich eine vorläufige Fassung des Artikels besprechen durfte und die mir wichtige Hinweise gegeben haben. Mein besonderer Dank gilt Hannes Amberger (Salerno) für das sorgfältige Korrekturlesen.

${ }^{2}$ Hier und im weiteren Verlauf werden die Quellenangaben bei den Gattungs- und Artnamen, im Gegensatz zu Verweisen auf das Literaturverzeichnis, mit dem Komma vor der Jahreszahl angegeben, wie es in der biologischen Nomenklatur üblich ist. Der Autor, der keine biologische Ausbildung hat, würde sich über Anregungen von Ornithologen besonders freuen.

${ }^{3}$ Für Beschreibungen sowie englische, französische, deutsche und spanische Trivialnamen der Arten, die der Familie Sylviidae angehören, s. die Einträge in der Online-Datenbank „Handbook of the Birds of the World Alive“ (Bairlein, Bonan 2018 zur Familie Sylviidae allgemein, mit Einträgen von anderen Autoren zu einzelnen Arten). Vgl. die deutschen Beschreibungen und Trivialnamen in Bezzel 1993, 267-404 (die Arten der Gattung Sylvia auf S. 328-364). Für die russischen Trivialnamen der Arten aus der Gattung Sylvia (mit Äquivalenten auf Latein, Englisch, Deutsch und Französisch) s. Boehme, Flint 1994, 343-345.

${ }^{4}$ Das Erscheinen des Buchstaben „y“ in der Wurzel lässt sich leicht erklären, nämlich als früher für korrekt gehaltene Schreibweise desselben Wortes (s. unten Abt. 4).

${ }^{5}$ James A. Jobling bietet zwar in seinem Wörterbuch der wissenschaftlichen Vogelnamen für einige Lexeme relativ umfangreiche Listen der Belege von Aristoteles bis Linné (wie etwa im Fall des Vogelnamens pyrrhulas, Jobling 2010, 327, der weiter unten behandelt wird, s. Anm. 15-17), beginnt aber seine Artikel Sylvia und sylvia, in denen er die Geschichte dieses Wortes als Vogelbezeichnung skizziert, erst mit Belegen aus dem 18. Jh. Als erste Beschreibung des sylvia genannten Vogels führt Jobling die von Carl von Linné an: „Motacilla supra cinerea, subtus alba, rectrice prima longitudinaliter dimidiato albo“ (Linné 1746, 85). Jobling erklärt die Herkunft des Wortes folgendermaßen: „Mod[ern] L[atin] sylvia woodland sprite, little bird, warbler ([derived from] L[atin] silva woodland)“. Die Deutung des neulateinischen sylvia als ,woodland sprite“ "Waldelfe' ist anscheinend eine unbegründete Vermutung von Jobling. Erweiterte Fassungen dieser Artikel Sylvia und sylvia sind auch in seinem neuen „Key to scientific names in ornithology" veröffentlicht (Jobling 2018a-b). Dort erscheint noch eine neue Bemerkung: „Roman myth[ology]. Rhea Silvia, mother of the twins Romulus and Remus, founders of Rome, goddess of the woods and associated with the River Tiber (Tanysiptera)" (Jobling 2018b). Die Herkunft des Vogelnamens sylvia bleibt für Jobling also unbekannt. Rhea Silvia ist nämlich mit dem Vogelnamen nur indirekt, die Waldelfe eher gar nicht verbunden (warum das Wort Tanysiptera, der Gattungsname der Paradieseisvögel, hier bei Jobling erscheint, ist mir unklar, denn weder dieses Wort, noch diese Vogelgattung hängt, meines Wissens, mit dem Wort und mit der Gattung silvia zusammen). Die Untersuchung der vorlinneischen Geschichte des Vogelnamens sylvia bleibt also bis jetzt anscheinend ein Desiderat. 


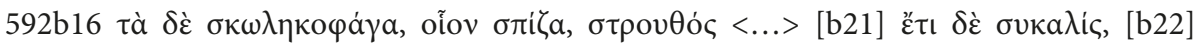

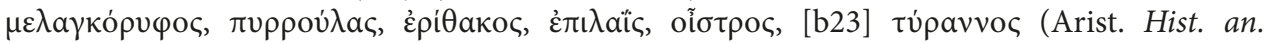
$592 \mathrm{~b} 16-23){ }^{6}$

In anscheinend allen gedruckten Editionen seit der princeps von Aldus Manutius bis zur letzten, die von David M. Balme vorbereitet und im Jahre 2002 von Allan Gotthelf

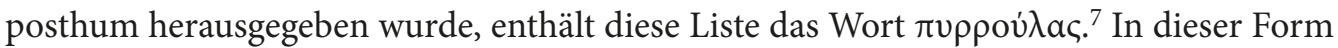
aber ist das Wort - ein Hapax legomenon - in keiner der bekannten Aristoteles-Handschriften belegt. Die in der Edition von Balme angegebenen Varianten sind folgende:

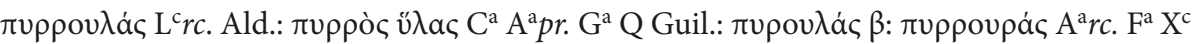
$\gamma$ (exc. $\left.L^{c} r c.\right){ }^{8}$

Die ursprüngliche Lesart des Mitte des 15. Jhs. entstandenen, Aldus' Edition zugrundeliegenden Kodex $\mathrm{L}^{\mathrm{c}}$, wie in den restlichen Kodizes der Familie $\gamma$, war $\pi v \rho \rho o v-$

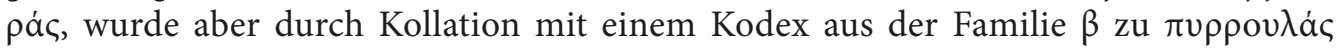
korrigiert. Aldus (und die weitere editorische Tradition nach seinem Beispiel) hat diese korrigierte Lesart übernommen. ${ }^{9}$ Dabei hat er, wohl als erster, die Verschiebung des Akzents willkürlich vorgeschlagen ( $\pi v \rho \rho o v \lambda a ́ \varsigma>\pi v \rho \rho o v ́ \lambda a \varsigma)$; diese in der Aldine bezeugte Verschiebung ist, wie wir sehen, in Balmes Apparat nicht berücksichtigt. Balme schreibt dem venezianischen Erstdrucker - anscheinend irrtümlich — die oxytonische Lesart

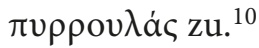

Ein Versuch, diese Stelle neu zu emendieren, fällt sicherlich nicht in den Gegenstandsbereich dieses Artikels. Abgesehen vom Akzent sehe ich keine gewichtigen Gründe, die Richtigkeit der seit Aldus üblichen Lesart zu bezweifeln. ${ }^{11}$ Die Überlieferung

${ }^{6}$ Nach der Edition Balme 2002, 342. „Andre Vögel fressen Würmer, zum Beispiel der Fink, der Sperling $\langle\ldots\rangle$. Dann gehören hierher Sykalis, die Sumpfmeise, Pyrrhulas, Erithakos, Epilaïs, Oistros und das Goldhähnchen“" (Aubert, Wimmer 1868, Bd. 2, 131).

7 Vgl. Manutius 1497, f. 107r; Camus 1783, t. 1, 468; Schneider 1811, t. 1, 355; Bekker 1831, Sp. 592b; Bussemaker 1864, 151; Dittmeyer 1907, 307; Louis 1969, 15; Balme 2002, 342. Die zahlreichen Ausgaben, die zwischen Aldus und Camus erschienen, habe ich nicht geprüft (ausgenommen die Ausgabe von 1619, s. unten Abt. 3).

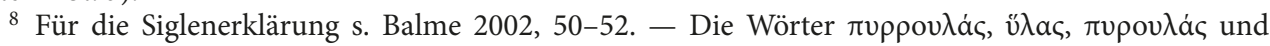

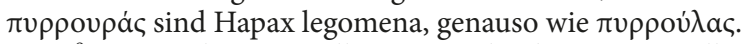

9 Der nach einer Kollation verschiedener Textquellen und wohl nach einer vom Kopisten selbst unternommenen Emendation geschriebene Kodex L L $^{c}$ (Ambr. I 56 sup.) war Antigraphon einer der Druckvorlagen von Aldus oder, obschon weniger wahrscheinlich, die Druckvorlage selbst (Sicherl 1997, 44-46; Balme 2002, 30-31, 36-38; Berger 2005, 155-158). In Friederike Bergers Monographie zur Überlieferungsgeschichte der Historia animalium ist $\mathrm{L}^{\mathrm{c}}$ mit einem anderen Sigel versehen — „“.

10 Manutius 1497, f. 107r. Balme hat für seine Edition eines der Exemplare aus der British Library (IB 24423), sowie ein anderes aus eigenem Besitz benutzt (Balme 2002, 36). Ich habe folgende digitalisierte online zugängliche Exemplare geprüft: Vatikan, Biblioteca Apostolica Vaticana, Stamp.Barb.CCC. V. 15 (https://digi.vatlib.it/view/Stamp.Barb.CCC. V.15); München, Bayerische Staatsbibliothek, BSB-Ink A-698 (http://daten.digitale-sammlungen.de/bsb00045770/image_221); Zürich, Zentralbibliothek Zürich, Ink K 257 (https://www.e-rara.ch/zuz/content/pageview/18758184). — D’Arcy W. Thompson erwähnt zwar

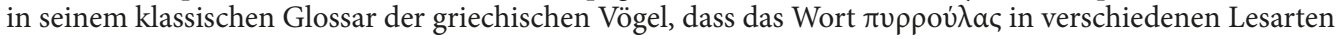

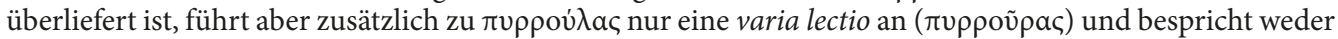
diese graphischen Schwankungen, noch die lateinischen Übersetzungen (Thompson 1895, 152).

11 Diese Lesart wird vielleicht von einem ähnlichen neugriechischen Vogelnamen gestützt (vermutet Thompson 1895, 152, ohne freilich den fraglichen Vogelnamen direkt anzugeben); das gilt allerdings nur in dem Falle, dass das neugriechische Wort wirklich im Volksmund überliefert wurde und nicht vom westlichen wissenschaftlichen Namen pyrrhula abhängig war - andernfalls verliert das neugriechische Wort für die Bewertung von 592b22 seine Argumentationskraft, weil man es dann mit einem Zirkelschluss zu tun 


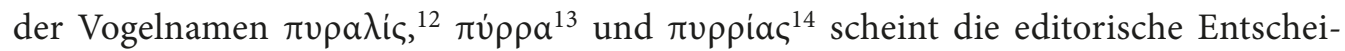
dung bei 592b22 weder unterstützen, noch widerlegen zu können. Ein Argument für die

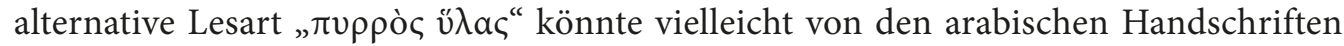
geliefert werden, aber um diese Möglichkeit bewerten zu können, muss man noch auf die Erscheinung der Edition des arabischen Textes warten (vgl. unten Anm. 23). Was die Etymologie des von Aldus rekonstruierten Wortes $\pi v \rho \rho o u ́ \lambda a \varsigma$ angeht, wird sie bei Pierre Chantraine im Artikel über das Adjektiv $\pi v \rho \rho o ́ \varsigma ~ ' f e u e r f a r b e n '{ }^{15}$ erläutert. Der Vogelname sei also mittels des „suffixe diminutif et expressif“ -ovג- vom Adjektiv abgeleitet (Chantraine 1999, 959). In den Wörterbüchern von Hjalmar Frisk und Robert

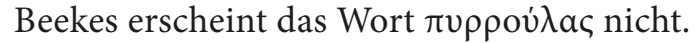

1544 hat der englische Naturforscher William Turner den ersten Versuch gewagt, zu den antiken Vogelnamen systematisch zeitgenössische volkssprachliche Entsprechungen

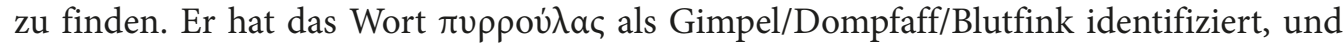
zwar nur aufgrund der Übereinstimmung zwischen der Farbe des Gimpels und der vermutlichen Etymologie des griechischen Wortes. ${ }^{16}$ Deshalb gilt Pyrrhula pyrrhula (Linnaeus, 1758) immer noch als wissenschaftlicher Name des Gimpels. ${ }^{17}$

Soweit zum Nachleben des Wortes $\pi v \rho \rho o u ́ \lambda a c$. Im Folgenden wird das Schicksal der

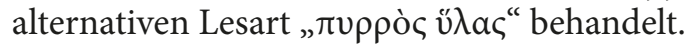

hat. Die von mir in diesem Zusammenhang versuchte Auseinandersetzung wäre hier unangebracht und wird später getrennt veröffentlicht.

12 Das Wort $\pi v \rho a \lambda i \varsigma$ (mit Varianten $\pi v \rho a \lambda \lambda i \varsigma, \pi v \rho \rho a \lambda i \varsigma$ ) mag sowohl von $\pi \tilde{v} \rho / \pi v \rho \rho o ́ \varsigma$, als auch von

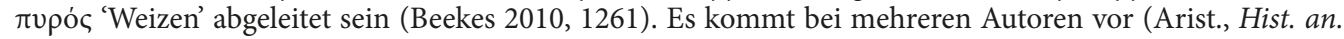
609a18-19; Callim. fr. 416 Pf. (100c4 Schn.); Ael. NA 5, 48, 10; vgl. Hsch. 4458) und bezeichnet wohl eine

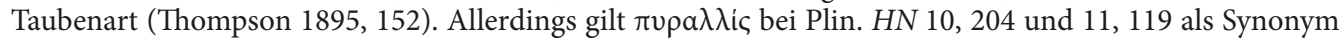
des Insektennamens pyrausta; Phylotimos ap. Orib. 2, 69, 9 bezeichnet mit $\pi v \rho a \lambda \lambda$ is eine Olivensorte; in einem auf Papyrus aus dem 1.-2. Jh. n. Chr. erhaltenen Rezept für die Lederfärbung (PSI inv. 22011, 54; vgl. Halleux 1981, 160-163) wird das Wort $\pi v \rho[\alpha] \lambda \lambda$ ic ebenfalls als Pflanzenname benutzt.

${ }_{13}$ Der Vogelname $\pi \dot{\rho} \rho \rho \alpha$ ist lediglich in Ael. NA 4, 5, 3 belegt, sowie in Manuel Philes' (13.-14. Jh.) Gedicht De proprietate animalium (Dübner, Lehrs 1862, 17, Z.36=Vers 685), das von Älian abhängig ist (Krumbacher 1897, 774). In diesen beiden Quellen steht nur, dass $\pi \dot{\rho} \rho \rho \alpha$ Feind der Taubenart $\tau \rho v \gamma \omega \dot{\omega} v$

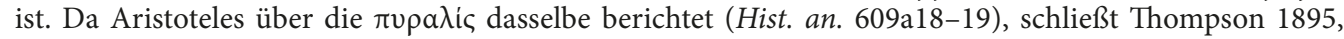
152 vernünftigerweise, dass $\pi \dot{v} \rho \rho a$ mit $\pi v \rho a \lambda i \varsigma$ identisch sein mag (vgl. bes. die alternative Schreibweise $\pi v \rho \rho a \lambda i \varsigma)$.

14 Zum Vogelnamen זvppiaৎ gibt es drei widersprüchliche Belegstellen: Alexander von Myndos ap. Ath. 2, 69, 3; Dionys. Per. Ixeut. 3, 13, 22 und Hsch. 4461. Die Auseinandersetzung damit hat mich genötigt, eine für den vorliegenden Artikel zu umfangreiche Abhandlung zu verfassen, die hier ausgespart werden kann. Was für den vorliegenden Aufsatz wichtig ist, sind die bei den drei Belegstellen überlieferten bzw.

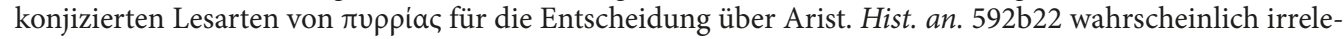
vant, denn in keiner Handschrift an 592b22 sind nach dem Rho Spuren des Jotas überliefert.

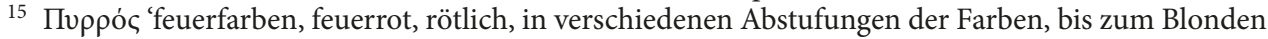
hin' (Pape 1880, 825); 'flame-coloured, yellowish-red; with red hair; tawny; red; blushing' (Liddell, Scott, Jones, 1940, s. v.); 'rougeâtre, roux' (Chantraine 1999, 959).

16 „Ego, nominis etymologiam secutus, rubicillam Anglorum bulfincam et Germanorum bloudvincam esse conjicio“" ("Meinerseits vermute ich, der Etymologie des Namens folgend, dass rubicilla der bullfinch der

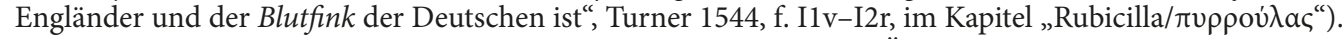
Das lateinische Wort rubicilla ist ein Neologismus aus Theodor Gazas Übersetzung der Historia animalium. Über diese Übersetzung s. unten Abt. 2. - Hier und im Weiteren wird die Interpunktion und Großschreibung in den Zitaten aus mittelalterlichen und frühneuzeitlichen Quellen normalisiert; die lateinischen und volkssprachlichen Tiernamen werden kursiv gedruckt; die frühneuzeitlichen Werke werden, wenn nicht anders bemerkt, nach der Erstausgabe zitiert.

17 Zur Systematik und Nomenklatur der Gimpel s.: Collar, Newton, Bonan 2018; Bezzel 1993, 600679 (zur ganzen Familie Fringillidae), sowie Boehme, Flint 1994, 436 (speziell zur Gattung Pyrrhula). 


\section{Lateinische Übersetzungen von Arist. Hist. an. $592 \mathrm{~b} 22$}

Bis jetzt hat anscheinend kein Forscher, der sich mit griechischen Vogelnamen befasst hat, das als alternative Lesart in Arist. Hist. an. 592b22 belegte Wort úlas studiert. Diese Lesart hatte aber in der frühen Neuzeit eine bis jetzt unbemerkte indirekte Rezeption. Die aristotelische „Tiergeschichte" wirkte nämlich auf die Entstehung der frühneuzeitlichen und modernen zoologischen Nomenklatur nicht nur direkt, sondern auch durch die lateinischen Übersetzungen (Beullens 2008; Vorobyev 2015). Wenn man unsere Textstelle 592b21-23 in den fünf existierenden Übersetzungen vergleicht, sieht man, dass wenigstens

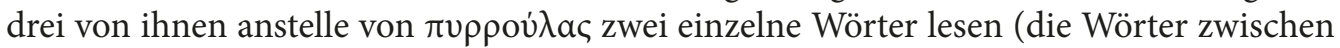

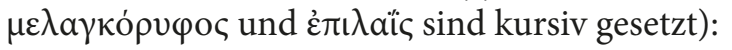

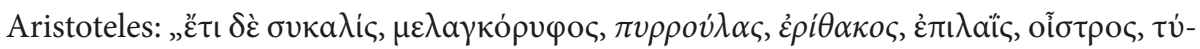
pavvoc" (Balme 2002, 342; vgl. oben Abt. 1).

1. Michael Scotus: „Et ex eo etiam sunt keokaloz, makanbeoroh, forozcheloz, autueche, aquila, cehostos, toranoz". 18

2. Wilhelm von Moerbeke: „Adhuc autem sikallis, melanokorifus, pyrrus, ylas, erythacus, epylis, ystrus, tyrannus".19

3. Georgios Trapezuntius: „Praeterea calida, ficedula, flavio, materio, erythacus, curruca, asillus, tyrannus“ ${ }^{20}$

4. Theodor Gaza: „Item ficedula, atricapilla, rubicilla, rubecula, silvia, curuca, asilus, tyrannus“.21

18 In den nächsten Jahren müsste eine kritische Edition von Scotus' Übersetzung der Hist. an. erscheinen, an der Aafke van Oppenraay arbeitet (https://www.huygens.knaw.nl/aristoteles-semitico-latinus-enavicenna-latinus). Als provisorische Textquelle wird üblicherweise der Text benutzt, den Christian Hünemörder durch die Kollation von drei Handschriften erstellt hat. Er wurde in Form eines von Benedikt Konrad Vollmann vorbereiteten Manuskripts wenigstens in einem Exemplar gedruckt, das in der Bayerischen Staatsbibliothek aufbewahrt wird (Hünemörder 1994). Ich durfte eine digitale Version davon benutzen, die mir Friederike Berger freundlicherweise zur Verfügung gestellt hat.

19 Zurzeit ist nur die kritische Ausgabe der ersten fünf Bücher der Historia animalium in Wilhelm von Moerbekes Übersetzung veröffentlicht worden (Beullens, Bossier 2000). Die restlichen Bücher sollen als Teil XVII.2.I.2 in der Reihe Aristoteles Latinus erscheinen. Mittlerweile ist eine elektronische Version, wenn auch ohne Apparat, in der Aristoteles Latinus Database (http://clt.brepolis.net/ald/Default.aspx) zugänglich, die für die vorliegende Studie benutzt wurde.

20 Diese Übersetzung wurde nie gedruckt. Ihr Text ist hier der Handschrift Florenz, Biblioteca Medicea Laurenziana, Plut. 84.9 (f. 107v) entnommen. Dieser Kodex enthält eigenhändige Korrekturen von Trapezuntius und gilt daher als wichtigste Kopie seiner Übersetzung (vgl. Monfasani 1984, 705-707). Ein Digitalisat davon kann unter http://mss.bmlonline.it/Catalogo.aspx?Shelfmark=Plut.84.9 konsultiert werden.

${ }^{21}$ So lautet sowohl der Text der 1476 gedruckten editio princeps (Gaza 1476, f. 1 8v-9r), als auch der des Prachtkodex Vat. lat. 2094 (f. 117r, abrufbar unter http://digi.vatlib.it/view/MSS_Vat.lat.2094), der Anfang der 1470er Jahre unter Gazas Betreuung kopiert und von ihm dem Papst Sixtus IV. geschenkt wurde (über diese zwei Textzeugen s. Monfasani 2006; Beullens, Gotthelf 2007, passim). Der einzige Unterschied besteht darin, dass die Druckausgabe attricapilla statt atricapilla liest, aber Schwankungen in der Schreibung von Doppelkonsonanten sind in diesem Druck sehr häufig. - Dieselbe Liste von Vogelnamen, mit einer zusätzlichen Erläuterung des Wortes atricapilla, befindet sich auch auf der Seite 45 der Handschrift Montecassino, Archivio dell'Abbazia, 649 (der Kodex ist paginiert): „Ficedula, atricapilla quae nigras h(ab)et plumas in vertice, rubicilla, rubecula, silvia, curuca, asylus, tirannus“ (Autopsie Februar 2018). Diese Handschrift ist eine wohl in der ersten Hälfte des 16. Jhs. geschaffene Kopie der verkürzten Fassung seiner Übersetzung, die Gaza Ende der 1460er oder Anfang der 1470er Jahre selbst vorbereitet und mit Kommentaren versehen hat. Die Schreibweise „asylus, tirannus“ statt „asilus, tyrannus“ dürfte von einem Kopistenfehler herkommen, weil die nächste Phrase (wenigstens für den zweiten Vogelnamen) eine korrekte Schreibung aufweist: „de forma et sp(eci)e tyranni aviculae“. Diese Handschrift ist bislang fast unerforscht geblieben (vgl. kurze 
5. Julius Caesar Scaliger: „Item ficedula, atriceps, byrriola, erithacus, curuca, asilius, tyrannus".22

Die erste lateinische Übersetzung wurde im zweiten Jahrzehnt des 13. Jahrhunderts von Michael Scotus angefertigt, wobei seine Vorlage kein griechischer Text war, sondern die arabische Fassung einer gekürzten Version von Hist. an. Es bleibt unklar, ob die arabische Vorlage von Scotus anstelle des griechischen Wortes $\pi v \rho \rho o v ่ \lambda a \varsigma$ ein einziges Wort oder zwei Wörter hatte (das überlieferte forozcheloz könnte auch zwei ohne Trennung geschriebene Wörter darstellen), ${ }^{23}$ wobei das nächste Wort, autueche, wahrscheinlich dem griechischen épí $\theta$ aкоৎ entspricht. ${ }^{24}$

In der nächsten Übersetzung, die Wilhelm von Moerbeke in den 1260er Jahren vornahm, und zwar bereits direkt aus dem Griechischen, stehen schon sicher zwei

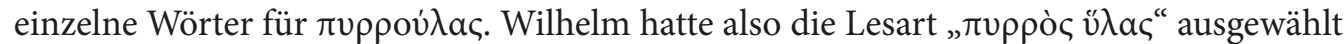
(über seine Vorlagen s. Berger 2005: 182-186) ${ }^{25}$.

Der Autor der nächsten, 1449-1450 entstandenen Übersetzung, der byzantinische Emigrant Georgios Trapezuntius, benutzte nicht nur einige griechische Handschriften als Vorlagen, sondern auch die Übersetzung des Wilhelm von Moerbeke (Dittmeyer 1902, 30; vgl. Monfasani 1984, 706). Indem Trapezuntius versuchte, seinen Text nach dem humanistischen Geschmack zu gestalten und einfache Transliterationen griechi-

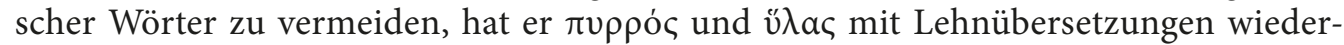

Nachrichten darüber in: Kristeller 1977, 342; Miglio 1978, XXVIII, XXXV n. 53, LXXI; Monfasani 1999, 239 n. 42). Die oben vorgeschlagene Datierung der Handschrift beruht auf einer Analyse der Schrift und insbesondere der Wasserzeichen (Meerjungfrau — sehr ähnlich Briquet 13900: Neapel, Imola, Fabriano 1533-1539; Löwe - ähnlich Briquet 10548: Neapel 1525, 1528). Ich beschäftige mich jetzt mit der Kollation dieser Handschrift aus Montecassino mit dem Vatikankodex und mit der editio princeps.

22 So in der editio princeps aus dem Jahre 1619 (Scaliger 1619, 885). 1811 wurde Scaligers Übersetzung zum zweiten Mal gedruckt, und zwar in der Bearbeitung von Johann Gottlob Schneider, in der unsere Stelle folgendermaßen lautet: „Item ficedula (sycalis), atriceps (Gazae atricapilla), pyrrhulas (Gazae rubecula, Scaligero byrriola), erithacus (Gazae silvia), curuca, asilus (oestrus), tyrannus" (Schneider 1811, T. II, 332).

23 Vielleicht wird die kritische Ausgabe von van Oppenraay zur Lösung dieses Problems beitragen. Die für den Herbst 2018 geplante Veröffentlichung der kritischen Edition des arabischen Textes, der für Scotus als Vorlage diente (Filius 2018), wird auch sicher hilfreich sein. Falls der arabische Text hier eindeutig

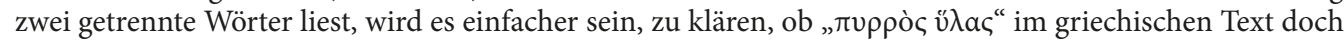
der allgemein akzeptierten Lesart $\pi v \rho \rho o u ́ \lambda a \varsigma$ vorgezogen werden muss (vgl. oben Abt. 1).

${ }^{24}$ Das Wort autueche wird an keiner weiteren Stelle in Scotus' Übersetzung erwähnt. Das ist aber

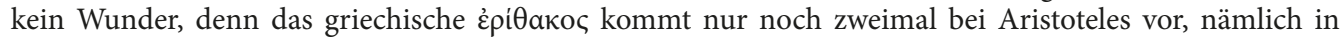
632b28 und 632b29, wobei dieser Textteil in Scotus' Übersetzung nicht eingeschlossen wurde. Deswegen scheint nichts der Identifizierung von autueche mit épi $\theta$ aко im Wege zu stehen. Wenn die arabische Ausgabe von Filius 2018 veröffentlicht wird, wird es möglich sein, diese Vermutung zu prüfen, sowie zu klären,

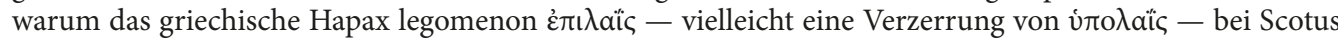
als aquila 'Adler' übersetzt wird, obwohl der Letztere offensichtlich nicht zu den wurmfressenden Vögeln zählt.

25 Die Kenntnis des in lateinischen Quellen gut belegten Eigennamens Hylas (z. B. Ov. Ars am. 2, 110; Verg. Ecl. 6, 44) hätte für einen mittelalterlichen oder humanistischen Gelehrten die Plausibilität der Lesart

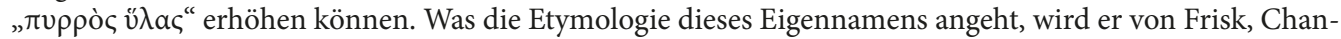
traine und Beekes im Artikel v́lá $\omega$ 'bellen' mit Bezug auf den Aufsatz von Paul Kretschmer erwähnt (Frisk 1960-1972, Bd. 2, 961; Chantraine 1999, 1154; Beekes 2010, 1529; Kretschmer 1925, 35-36). Falls die Lesart

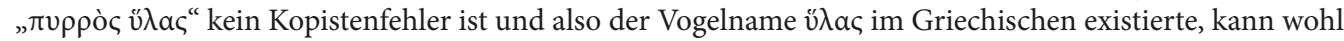
seine Herkunft wie die des homonymen Eigennamens erklärt, d. h. von v́láw abgeleitet werden, vielleicht mit Bezug auf die bellende Stimme des Vogels. 
gegeben, nämlich flavio und materio (von flavus und materia abgeleitete Neubildungen). ${ }^{26}$

Theodor Gaza, ein anderer griechischer Gelehrter, der um 1473-1474 die nächste Übersetzung abschloss, hat zwar dem Text von Trapezuntius viel entnommen, versuchte aber die Entlehnungen zu modifizieren, sodass sie wie von ihm selbst eingeführte Wörter aussahen. Unter den von Gaza benutzten griechischen Handschriften gab es (wenigstens)

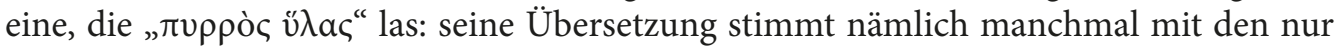
in den Kodizes $A^{a}$ und $C^{a}$ bezeugten Lesarten überein (Dittmeyer 1902, 34; vgl. Berger 2005: 144). Jedenfalls aber folgte er auch dem Beispiel von Trapezuntius „flavio materio“. Er verwendete nur eine andere lateinische Wurzel mit der Semantik der feuerroten bzw.

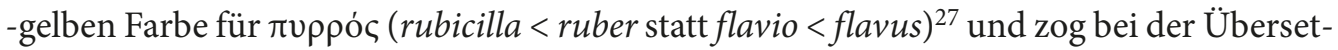
zung von ü $\lambda a \varsigma$ der Bedeutung von ư $\lambda \eta$ 'Stoff, Holz' die andere, 'Wald, Gehölz', vor (silvia

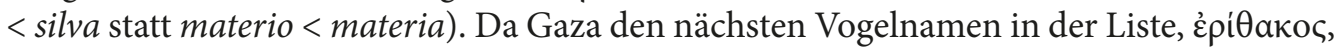
als rubecula übersetzte, ${ }^{28}$ veränderte er die Wortfolge, sodass seine rubecula und rubicilla

${ }^{26}$ Diese von Trapezuntius eingeführten Neologismen der dritten Deklination bilden anscheinend die in den antiken Quellen bezeugten Tiernamen nach (asio/axio, papilio, rubellio, stellio, vespertilio usw.). Die Tatsache, dass dasselbe Wortbildungsmuster für die beiden Neologismen verwendet wurde, könnte darauf

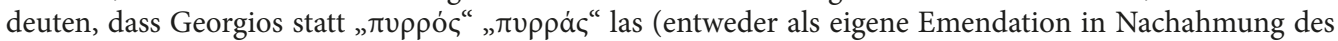
benachbarten ưlac, oder als eine heute nicht mehr überlieferte handschriftliche Variante). Weitere Vogel-

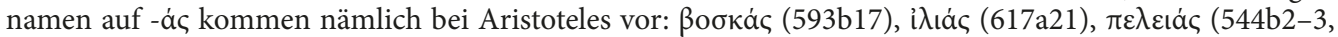

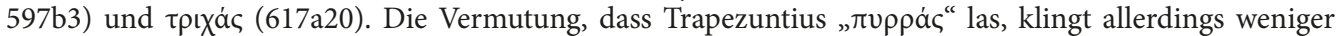
überzeugend, hält man sich diese letzteren Wörter in Trapezuntius' Übersetzung vor Augen. Er gibt sie nämlich mit verschiedenen Mitteln wieder, nur nicht mit dem Suffix -io: basca (Laur. Plut. 84.9, f. 108v; vielleicht fehlerhaft statt bosca); ylias (f. 133v); pelias (f. 112v) und palumbes (f. 65r); plumaria (f. 133v). - In der zitierten Vogelnamenliste aus Trapezuntius' Übersetzung verwundert noch eine weitere Besonderheit. Hier

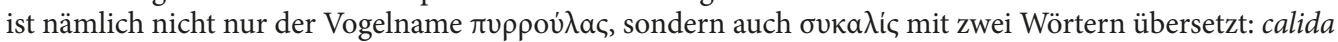
und ficedula. Ficedula ist ein in der Antike bezeugtes Wort und ein offensichtliches Äquivalent des griechi-

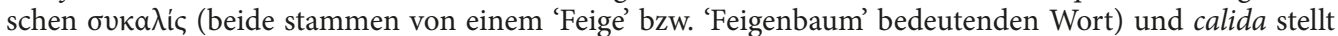
einen Versuch dar, die griechische Lesart кa $\lambda$ ic zu übersetzen, die statt $\sigma v \kappa \alpha \lambda i$ ic in drei Handschriften belegt ist (Balme 2002, 342). D. h., entweder hatte der Kopist einer der von Trapezuntius benutzten griechischen Handschriften einen verbreiteten Fehler begangen, indem er die alternative Lesart, die als Rand- oder Interlinearglosse in seiner Vorlage stand, beim Kopieren direkt in den Text hinzugefügt hatte, oder Trapezuntius hielt bei der Kollation der griechischen Handschriften zwei Lesarten aus verschiedenen Vorlagen für gültig und übersetzte beide nacheinander. Das Letztere würde allerdings für eine zu niedrige Qualität seiner textkritischen Methode sprechen, daher würde ich den vermutlichen Kopistenfehler vorziehen.

27 Theoretisch könnte man annehmen, dass silvia ein paläographisch einfach erklärbarer Kopistenfehler statt des richtigen fulvia ist. Das letztere Wort wäre dann ein von fulvus 'rotgelb' abgeleiteter Neologismus, der dem griechischen $\pi v \rho \rho o v ́ \lambda a \varsigma$ entsprechen könnte. In der Tat ist aber diese Konjektur äußerst unwahrscheinlich, da alle drei primären Textzeugen (der Kodex Vat. lat. 2094, die editio princeps und die Handschrift aus Montecassino) hier deutlich silvia lesen. - Was rubicilla angeht, könnte dieses Wort des-

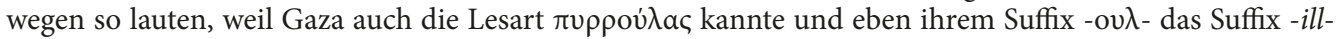
gleichsetzte, wie es in Vorobyev 2015, 163, Anm. 13 vermutet wird. Jetzt würde ich aber eine ökonomischere

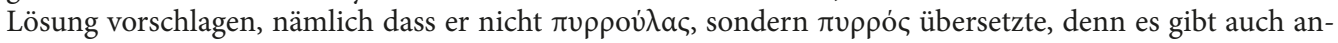

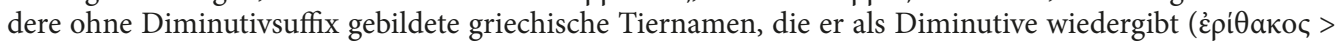

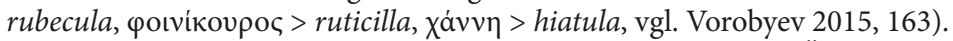

28 Rubecula ist ein Neologismus von Gaza, den er für die Übersetzung des Wortes épi $\theta$ ako benutzt, und zwar nicht nur hier, sondern auch an den beiden anderen Stellen in der Hist. an., wo dieser Vogelname

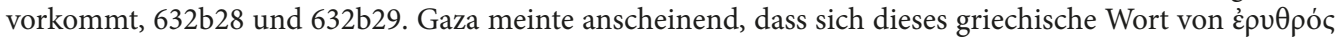
'rot' ableite. Ob diese Etymologie stimmt, bleibt unklar. Sundevall findet sie plausibel, da er diesen Vogelnamen als Zusammensetzung der Wurzel غ̇ $\rho \theta \rho-$ mit dem Wort $\theta \tilde{a} \kappa o \varsigma$ 'Sitz' betrachtet, wobei er das letztere Bestandteil in diesem Fall als 'Gesäß, Hinterteil' deutet (Sundevall 1863, 110-111). Thompson äußert sich gegenüber dieser Etymologie kritisch (Thompson 1895, 57). Frisk behandelt im Rahmen des Eintrags

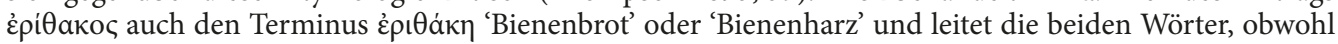


nacheinander stehen (wohl damit die Liste einen „wohlklingenden“ oder „logischeren“

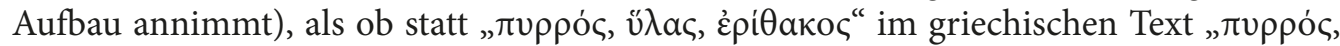

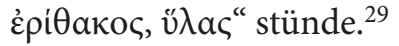

Während die Übersetzungen von Scotus, Moerbeke und Trapezuntius nie gedruckt wurden, wurde der Text von Gaza mehrmals aufgelegt und übte vom 15. bis zum 18. Jh. einen außerordentlichen Einfluss aus (Perfetti 1995, 257-260; Monfasani 1999, 214-217; Beullens, Gotthelf 2007, 469-470 u. 503-505). Einige der von ihm eingeführten Neologismen wurden in der frühneuzeitlichen Naturwissenschaft weithin benutzt und sind sogar in der heutigen zoologischen Nomenklatur als Gattungs- bzw. Artnamen gültig (Vorobyev 2015). Das ist auch bei rubecula (Vorobyev 2015, 163) und, wie es sich jetzt erwies, silvia der Fall. In Vorobyev, 2015 wird das moderne Nachleben nur von Gazas Neologismen stricto sensu betrachtet, d. h. der sogenannten „neologisms of form“. Zu den Neologismen Gazas zählen aber auch die sogenannten „neologisms of sense“, d. h. die Wörter, die in antiken Quellen schon vorkommen, aber erst von Gaza in einer neuen Bedeutung, als Tierbezeichnungen, benutzt werden. ${ }^{30}$ Das das griechische ü $\lambda a \varsigma$ wiedergebende Wort silvia gehört eben den "neologisms of sense“ von Gaza. Er war der erste, der es als Vogelnamen verwendete, wobei der hohe Bekanntheitsgrad des Eigennamens Silvia dafür sorgte, dass diese neueingeführte Vogelbezeichnung den Geschmack der humanistischen Puristen nicht störte.

Eine neue Übersetzung der „Historia animalium“ wurde von Julius Caesar Scaliger in den 1530er Jahren abgefasst. Obschon er im Kommentar zu seiner Übersetzung Gaza stark kritisierte, konnte er die etablierte Autorität des byzantinischen Humanisten nicht erschüttern. Scaligers Text wurde nämlich posthum im Jahre 1619 gedruckt, und zum zweiten Mal erst im Jahre 1811 (Monfasani 1999, 216; vgl. oben Anm. 22). Deswegen hatte die Übersetzung byrriola, die Scaliger für $\pi v \rho \rho o u ́ \lambda a \varsigma$ vorschlug (Scaliger 1619, 885), keinen wesentlichen Einfluss. ${ }^{31}$

\section{Die frühneuzeitliche Rezeption und der Bedeutungswandel des Wortes silvia/sylvia}

Was war nun das Nachleben des Hapax legomenon silvia aus der Übersetzung von Theodor Gaza? Offenbar hat keiner der frühneuzeitlichen Zoologen verstanden, dass das

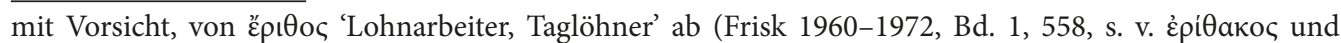
ع́pı $(\theta)$ ). Chantraine wiederholt zwar Frisks Hypothese, aber staunt über die Gründe dieser Wortbildung

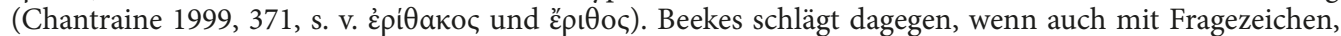
eine Herkunft aus dem vorgriechischen Substrat vor (Beekes 2010, 458).

29 Dass Gaza den aristotelischen Text weitgehend willkürlich veränderte, ist gut bekannt, vgl. Perfetti 1995, 261-280.

30 Diese Gruppe von Tiernamen in Gazas Übersetzung zählt 80 Wörter (nicht 66, wie in meiner vorläufigen Studie steht: Vorobyev 2015, 160). Zum Begriff „,neologism of sense“ s. Helander 2014, 37; Ramminger 2014, 29.

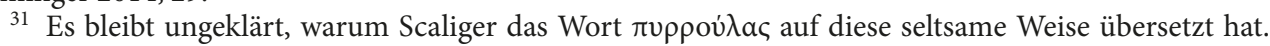
Es ist eher unwahrscheinlich, dass er das griechische Wort mit irgendeinem Vogelnamen, den er aus einem italienischen oder französischen Dialekt kannte (vgl. das französische bouvreuil 'Gimpel'), zu verbinden

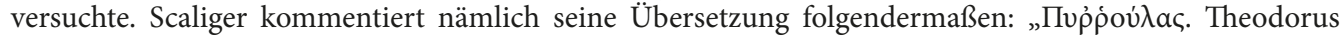
rubecula. Nos byrriolam ad verbum. Itali vocant petto rosso. Galli gorge rouge. Avis nota autumno, pinguis et

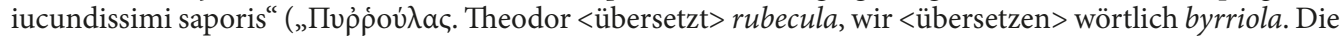
Italiener nennen <ihn> petto rosso, die Franzosen gorge rouge. Ein im Herbst anzutreffender Vogel, fett und von sehr angenehmem Geschmack“, Scaliger 1619, 885). Der Buchstabe „"“ kann auch kein Druckfehler sein, da das Wort auf der Seite zweimal auftaucht: in der Übersetzung und im Kommentar. 
Wort in Gazas Text wegen einer verderbten Lesart in der griechischen Handschriften-

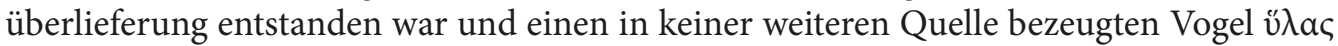
bezeichnete.

Der oben erwähnte William Turner hat 1544 vorgeschlagen, den aristotelischen Vogelnamen épi $\theta$ aкоৎ (und somit das von Gaza eingeführte lateinische Äquivalent rubecula) als Bezeichnung für das Rotkehlchen zu deuten. ${ }^{32}$ Da das Wort sylvia (über die Schreibung mit „y“ s. unten Abt. 4) bereits wenigstens seit der 1508 erschienenen Ausgabe von Erasmus' "Adagia“ als Synonym von rubecula galt, ${ }^{33}$ hat folglich auch das Wort sylvia die Bedeutung 'Rotkehlchen' übernommen. ${ }^{34}$

Der Schweizer Universalgelehrte Konrad Gessner hat im 1555 gedruckten ornithologischen Band seiner „Tiergeschichte“, des grundlegenden Werks der frühneuzeitlichen Zoologie, ${ }^{35}$ diese beiden Gleichsetzungen („rubecula = 'Rotkehlchen" laut Turner; „rubecula = sylvia“ laut Erasmus) übernommen. ${ }^{36}$ Er schrieb nämlich im Kapitel über das Rotkehlchen:

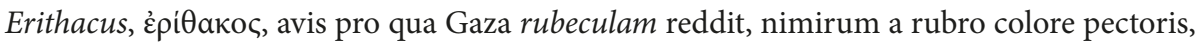
alibi vero sylviam (forte quod in sylvis degere soleat per aestatem) (Gessner 1555, 697). ${ }^{37}$

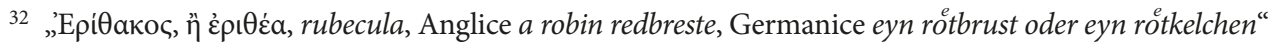
(Turner 1544, f. H 8r). Turner stützte sich bei dieser Identifikation wohl auf Niccolò Perottis „Cornucopiae“, wo

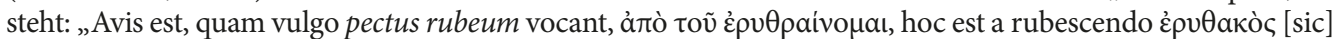

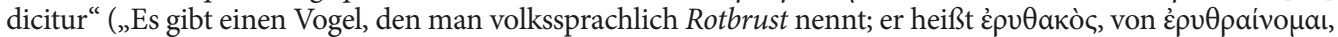
d. h. vom Rotsein", Perotti 1489, f. 103v), wobei sich der volkssprachliche Name pectus rubeum offensichtlich auf das italienische pettirosso 'Rotkehlchen' bezieht. - Der Vogelname silvia/sylvia taucht bei Turner nur außerhalb des Haupttextes auf, nämlich im Anhang, der Exzerpte aus Theodor Gazas Aristoteles-Übersetzung enthält, die allgemeine Informationen über Vögel liefern. Dort wird auch eine Liste der sich von Würmern und Insektenlarven ernährenden Vögel angeführt, die sich auf unsere Textstelle 592b16-23 und auf andere einschlägige Stellen stützt: „Vermibus aut ex toto, aut magna ex parte victitantes: fringilla, passer, rubetra, luteola et pari omnes, ficedula, atricapilla, rubicilla, rubecula, sylvia, curuca, asylus, florus, montifringilla, regulus et frugilega" (Turner 1544, f. K 2v). Über Turner s. wenigstens: Bäumer 1991, 333-335.

33 „Hanc [sc. erithacum avem] Theodorus Gaza nunc sylviam, nunc rubeculam interpretatur", bezüglich des Spruches „Unicum arbustum haud alit duos erithacos“ („Theodor Gaza übersetzt ihn [sc. den Vogel erithacus] bald als sylvia, bald als rubecula“, Erasmus 1508, f. 126r; die erste, weniger umfangreiche, Fassung von 1500 habe ich nicht geprüft). Weder Erasmus, noch die späteren Autoren haben nämlich bemerkt, dass

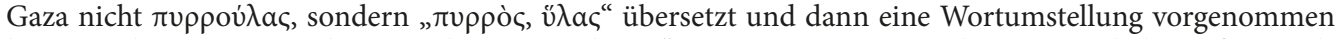
hat (man kann sogar noch im „Index Aristotelicum“ von Hermann Bonitz lesen, Gaza habe épiӨakoৎ als „sylvia et rubecula“ übersetzt, Bonitz 1870, 286).

${ }^{34}$ Diese Gleichsetzung von Erasmus wurde auch in der Lexikographie rezipiert. In Antonio de Nebrijas Wörterbuch gab es ursprünglich keinen Artikel silvia (Nebrija 1514, f. CXVIIIr-v; die Erstausgabe von 1492 blieb mir unzugänglich), aber in einer erweiterten Auflage von 1545 erscheint bereits der folgende Eintrag: „Silvia: avis est, quae et rubecula. G<raece> erithacos“ („Silvia ist derselbe Vogel, wie rubecula. Auf Griechisch erithacos", Nebrija 1545, f. x 3r).

35 S.wenigstens Friedrich 1995. Zum ornithologischen Band von Gessners Werk s. Springer, Kinzelbach 2009.

${ }^{36}$ In seinem früheren Werk, dem bibliographischen Kompendium „Pandecta““ (s. darüber z. B. Sabba 2012; Sergeev 2008, 529-530; Sergeev 2018, 81-82), das 1548 erschien, hatte Gessner diese Identifikationen von Erasmus und Turner noch nicht rezipiert. In der Abteilung der „Pandectae“ nämlich, die der Bibliographie zu einzelnen Tieren gewidmet ist, gesteht Gessner im Eintrag „silvia“, dass er kein griechisches Äquivalent dazu kennt: „Silvia vermiculis vescitur — haec verba in Theodori Gazae ex A<ristotelis $>$ Animalium historia traductione leguntur. Ego in Graeco textu, quod silviae Latino respondeat, vocabulum nullum invenio“ („Silvia ernährt sich von Würmern - diese Worte stehen in Theodor Gazas Übersetzung aus der Historia animalium von Aristoteles. Ich finde im griechischen Text kein Wort, das dem lateinischen silvia entsprechen würde"), Gessner 1548, f. 227r.

37 „Erithacus, દ́piӨ Farbe der Brust, wiedergibt, an einer anderen Stelle aber als sylvia (vielleicht weil er während des Sommers 
Der Autor der nächsten großen Tierenzyklopädie, Ulisse Aldrovandi, wiederholt im Kapitel „De erithaco, sive rubecula“ des zweiten Bandes seines 1600 veröffentlichten ornithologischen Buches fast wörtlich Gessners Meinung. ${ }^{38}$ Auch Jan Jonston teilt in seiner 1650 gedruckten „Historia naturalis animalium“ mit, dass sylvia eine alternative Übersetzung des Wortes épi $\theta$ ako sei. ${ }^{39}$

Das Wort silvia/sylvia hat sich gegen andere Bezeichnungen für das Rotkehlchen in der Konkurrenz nicht durchsetzen können. Es wurde nur selten in einigen Wörterbüchern und in wenigen zoologischen Schriften des 16.-18. Jhs. als einer der lateinischen Namen des Rotkehlchens erwähnt: ${ }^{40}$ die Hauptbezeichnung war das Wort rubecula und manchmal das aus dem Griechischen transliterierte erithacus. Das 1668 erschienene „Onomasticon zoicon" von Walter Charleton beispielsweise bietet das Wort rubecula als Hauptbezeichnung für das Rotkehlchen und das Wort erithacus als sein einziges lateinisches Synonym (Charleton 1668, 91). In dem einflussreichen ornithologischen Nachschlagwerk von Francis Willoughby, das John Ray posthum veröffentlicht hat (Bäumer 1996, 89-90), ist der Artikel über das Rotkehlchen „Rubecula sive Erithacus“ betitelt, wobei das Wort silvia/sylvia gar nicht erwähnt wird (Willoughby 1676, 160-161; vgl. auch den Index). ${ }^{41}$

Der von Gaza eingeführte Neologismus silvia wäre von den Zoologen vergessen worden und ein bloßes lexikographisches Kuriosum geblieben, aber die im 18. Jh. unternommenen Versuche, die Tiervielfalt nach der einen oder anderen Klassifikation zu systematisieren, setzten immer neue Umstellungen der bereits beschriebenen Tiergruppen, sowie die Einführung von neuen Taxa, voraus. Diese neuen Taxa brauchten neue Bezeichnungen, sodass die üblichen lateinischen Namen nicht ausreichten und bereits halbvergessene rare Wörter wiederverwertet wurden. So schlug 1750 Jacob Theodor Klein, ein Gegner

in den Wäldern zu leben pflegt)“. Auf der S. 699 werden dann entsprechende Namen auf verschiedenen Volkssprachen aufgeführt, die alle 'Rotkehlchen' bedeuten. Gessner hat die Information über das sommerliche Waldhabitat von sylvia, die der rubecula gleich sei, dem Traktat von Turner entnommen, der seine eigenen Beobachtungen über Rotkehlchen äußerte: „Rubeculae in aestate, ubi in sylvis satis superque alimenti suppetit, nec ullo infestantur frigore (quae res cogit illas in hyeme ad urbes, oppida et pagos confugere) cum prole ad desertissima quaeque loca secedunt“ („Die Rotkehlchen ziehen sich im Sommer — wenn es in Wäldern mehr als zu viel Futter gibt und sie von keiner Kälte gefährdet werden (eben dieser Umstand zwingt sie im Winter dazu, in große und kleine Städte sowie in Dörfer zu flüchten) - zusammen mit der Brut zu ganz öden Orten zurück“), Turner 1544, f. I 1r.

38 „Theodorus Gaza Aristotelis interpres alibi rubeculam reddidit, nimirum a pectoris rubedine, alibi etiam sylviam, forte quod in sylvis degere soleat per aestatem“ („Theodor Gaza, der Übersetzer von Aristoteles, hat <sie > mal als rubecula wiedergegeben, zweifelsohne wegen der roten Farbe der Brust, mal als sylvia, vielleicht weil sie während des Sommers in den Wäldern zu leben pflegt“, Aldrovandi 1600, 741). Über Aldrovandi s. wenigstens Riedl-Dorn 1989; Bäumer 1991, 74-119.

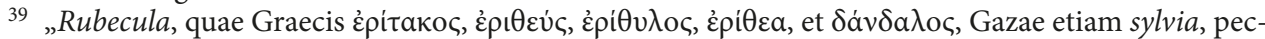

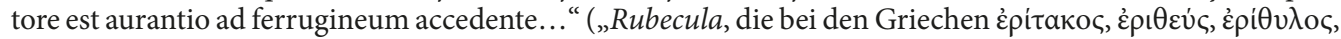

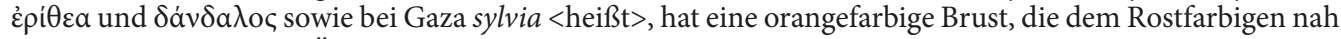
ist", Jonston 1650, 121). Über Jonston s. wenigstens Bäumer 1991, 131-149.

40 Z. B. im „Nomenclator“ von Hadrianus Junius (Junius 1567, 70; vgl. z. B. auch eine spätere Ausgabe: Junius 1620, 60, hier seltsamerweise auch mit rubellio gleichgesetzt), im Synonymwörterbuch von Francesco Serra (Serra 1654, 126) oder im Werk über die Naturgeschichte Polens von Gabryel Rzączyński (Rzączyński 1721, 279; Rzączyński 1745, 418).

${ }^{41}$ Darüber hinaus wird in so vollkommen verschiedenen Werken wie in Athanasius Kirchers „Arca Noe“ (Kircher 1675, 93), im verbreiteten Schulbuch „Orbis sensualium pictus“von Johann Amos Comenius (Comenius 1658, 45) oder in der wichtigen Quelle guter Vogelbilder, Eleazar Albins „Natural history of birds" (Albin 1731, 49), jeweils nur ein einziger lateinischer Name des Rotkehlchens angegeben, nämlich rubecula (bei Albin 1731 ist das fehlerhaft gedruckte rubeculus in der Errata-Liste auf der S. 100 zu rubecula korrigiert). 
des Systems von Linné (Geus 1977, 741), seine eigene Vogelklassifikation vor. Er teilte Vögel in Familien, dann in Gattungen und dann in Tribus. In der Gattung Nr. VII, die zur Familie Nr. IV gehörte, führte Klein eine Tribus ein, die er mit der Nr. III und dem lateinischen Namen Sylvia versah. Als Merkmale dieser 28 Arten zählenden Tribus führte er gewisse Besonderheiten der Brustform und des Brustgefieders auf. ${ }^{42}$ Die erste Art, die Klein in dieser Tribus beschreibt, heißt Sylvia sylvatica. Er gab für diese Art eine Reihe von volkssprachlichen Bezeichnungen an, sowie den üblichen lateinischen Namen rubecula. Auf diese Weise hat Klein das halbvergessene Wort sylvia in die zoologische Nomenklatur zurückgebracht, immer noch mit der Bedeutung 'Rotkehlchen' (Klein 1750, 14, 77-80).

Gegen Kleins Klassifikation setzte sich das linneische System durch. Gemäß der klassischen zehnten Auflage des „Systema naturae“ von Linné, die als Ausgangspunkt der modernen binären Tiernomenklatur gilt, gehören alle Vogelarten, die zu Kleins Tribus Sylvia zählten, zu Linnés Gattung Motacilla. Linné benutzte das Wort sylvia auch, aber nur als Artname: Motacilla sylvia (Linné 1758, 185).

Der Prozess einer Umordnung der Taxa ging weiter (und wird von den Biologen auch heute intensiv fortgeführt). So schlug der bereits erwähnte Naturforscher Giovanni Antonio Scopoli vor, den Umfang von Linnés Gattung Motacilla einzuschränken, indem er einige Arten daraus ausschloss und in eine neue Gattung gruppierte. Er nannte diese Gattung eben Sylvia (Scopoli 1769, 154-161). ${ }^{43}$ Warum gab Scopoli seinem neuen Taxon diesen Namen? Er entnahm ihn offensichtlich nicht Linnés „Systema naturae“, wo sylvia nur als Artname auftaucht, sondern aus Kleins Klassifikation. Genauso wie Klein rechnete Scopoli der Gattung Sylvia die Art Rotkehlchen zu: sie hieß in seiner Klassifikation Sylvia rubecula (Scopoli 1769, 156-157).

Später wurde der Umfang von Scopolis Gattung Sylvia weiter reduziert, sodass in ihr nur die Grasmücken verblieben, während die verschiedenen Rotkehlchen und einige andere Arten als andere selbständige Gattungen gesondert wurden. George Cuvier nämlich hängte dem 1800 erschienenen ersten Band seiner „Leçons d'anatomie comparée“ Tabellen an, in denen er eine neue Klassifikation des Tierreichs vorschlug. Die Tabelle, die der Vogelklassifikation gewidmet ist, enthält ein Taxon Motacilla, das in fünf auf Latein und Französisch bezeichnete Gattungen unterteilt ist: Silvia (fauvettes 'Grasmü-

${ }^{42}$ Es ist unbekannt, welcher direkten Quelle Klein das lateinische Wort entnommen hat; in seinem dreiseitigen „Indiculus autorum“ (Klein 1750, f. b3 1r-2r) werden u. a. Turner, Gessner, Aldrovandi und Jonston genannt, die das Wort sylvia erwähnten, sowie Gazas Aristoteles-Übersetzung. Es ist allerdings nicht ausgeschlossen, dass Kleins unmittelbare Quelle für das Wort sylvia ein späterer Text gewesen sein konnte. - Klein bot auch einen deutschen Namen dieser Tribus, nämlich Brustwenzel. Das ist wahrscheinlich ein von ihm eingeführter Neologismus (vgl. Adelung 1793, 1231).

${ }^{43}$ In seinem Vorwort teilt Scopoli mit, worin seine Klassifikation von denen von Linné und Klein abweicht, und spricht $u$. a. von der neuen Gattung Sylvia: „Ornithologiae Linnaeanae ordines et genera retinui, quorum fundamentum sunt rostrum, lingua, pedes; sed haec ipsa principia me coegerunt, ut genera nova Uriae, Tracheliae, Sylviae conderem, Kleiniana Brandae et Ploti revocarem, aliorumque caracteres [sic] ubique emendarem, ut paulo firmiore stet talo scientia“ („Ich habe die Ordnungen und Gattungen der linneischen Ornithologie beibehalten, deren Basis < die Eigenschaften> des Schnabels, der Zunge < und> der Füße bilden; aber genau diese Prinzipien haben mich gezwungen, neue Gattungen einzuführen (Uria, Trachelia, Sylvia), die kleinschen Gattungen Branda und Plotus abzuschaffen und überall die Merkmale anderer $<$ Gattungen $>$ zu korrigieren, sodass $<$ diese $>$ Wissenschaft auf etwas festeren Grundlagen steht", Scopoli 1769, f. A 3r-v). - Noch zu untersuchen bleibt die Herkunft des Artnamens sylvia bei einer Libelle und einem Schmetterling: Libellula sylvia (Fourcroy \& Geoffroy, 1785) und Papilio sylvia (Cramer, 1775). 
cken'), Erithacus (rouge-gorges 'Rotkehlchen'), ${ }^{44}$ Ficedula (figuiers), ${ }^{45}$ Regulus (roitelets 'Goldhähnchen') ${ }^{46}$ und Motacilla (hochequeues) ${ }^{47}$ (Cuvier 1800, tableau 2) ${ }^{48}$. Auf diese Weise ist das Wort silvia/sylvia, das seit Gessner für eine Bezeichnung des Rotkehlchens gehalten wurde, 1800 zur Bezeichnung der Gattung „Grasmücken“ geworden und wird immer noch ${ }^{49}$ als solche verwendet.

\section{Silvia oder sylvia?}

Es bleibt, eine triviale Frage zu beantworten, nämlich über die Gründe der Schreibungsschwankung silvia/sylvia. Es schien mir vernünftig, dieses rein graphische Problem von dem Problem der Identifikation und der Nomenklatur getrennt zu behandeln.

Das Wort Silvia ist als Eigenname gut bekannt, der sich von silva 'Wald' ableitet (Ernout, Meillet 2001, 626, Silvius s. v. silva). Heute ist es üblich, das Wort silva und seine Derivate mit „i“ zu schreiben, aber das war sogar noch im 18. Jh. nicht der Fall. In den Wörterbüchern und Werken zur Sprachpflege wurden Argumente angeführt, die die angebliche Korrektheit ${ }^{50}$ der Schreibweise sylva widerlegten. So schreibt 1744 der Verfasser eines der einflussreichsten „Antibarbari“ (Werke, in denen Barbarismen verzeichnet und bekämpft wurden), Johann Friedrich Nolte:

Silva per $I$, ex consensu antiquitatis, et librorum veterum. $<\ldots>$ Inde nomen Silvanus, Silvius, Silvia, frequentia in inscriptionibus et cod<icibus $>$ vet<eribus $>$ semper per $I$ Latinum. ${ }^{51}$

${ }^{44}$ Das Taxon Erithacus (Cuvier, 1800) ist immer noch gültig, vgl.: Boehme, Flint 1994, 304; Collar 2018.

45 Dieses Taxon von Cuvier lässt sich mit keinem deutschen taxonomisch gültigen Namen übersetzen. Der französische Name figuier wurde offensichtlich von Mathurin-Jacques Brisson als Übersetzung des lateinischen ficedula eingeführt (Brisson 1760, XLVI, 482-531 passim).

46 Das Taxon Regulus (Cuvier, 1800) ist immer noch gültig, vgl.: Boehme, Flint 1994, 342; Martens, Päckert 2018.

47 Der akzeptierte französische Trivialname der heute gültigen Gattung Motacilla (Linnaeus, 1758) 'Stelzen' ist bergeronnettes (Boehme, Flint 1994, 276; Tyler 2018).

${ }^{48}$ In seiner früheren, 1798 veröffentlichten Schrift, die speziell der Klassifikation von Tieren gewidmet ist, hatte Cuvier diese Unterteilung in Gattungen noch nicht durchdacht, indem er alle entsprechenden Arten der Gattung Motacilla zurechnete (Cuvier 1798, 218-221).

${ }^{49}$ Die Systematik wandelt sich permanent, sodass es z. B. im 19. Jh. Versuche gab, die Gattungen der Sperlingsvögel neu umzuordnen und den Umfang von Sylvia wieder zu ändern (vgl. Stresemann 1975, 108). Die Gattung Sylvia ist jedoch seit Cuvier als „Grasmücken“ gültig geblieben (Bezzel 1993, 328-364).

${ }^{50}$ Vgl. einige frühneuzeitliche Ausgaben von Statius' „Silvae“, wo der Titel mit „y“ geschrieben ist (z. B. Veenhusen 1671; Statius 1778). Der Titel der "Silva in scabiem“ von Angelo Poliziano wird in den modernen Editionen, die der Schreibweise des einzigen überlieferten Textzeugen folgen, auch mit „y“ geschrieben (Perosa 1954, Orvieto 1989).

51 „Silva < wird > mit I < geschrieben>, gemäß dem einhelligen Urteil der Antike und der alten Bücher. $<\ldots>$ Daher <wird $>$ der Name Silvanus, Silvius, Silvia beim häufigen Vorkommen in alten Inschriften und Kodizes immer mit dem lateinischen I <geschrieben> " (Nolte 1744, col. 166 s. v. silva). Vgl. seinen Artikel über den Buchstaben „y“: „Y littera Graeca est, quae, sicuti aliae Graecae $\theta, \kappa, \varphi, \chi, \zeta$, in iis tantum verbis est respicienda, quorum manifesta ex Graecis petita est derivatio, non a conjectura nugacium Grammaticorum. $\langle\ldots\rangle$ [V]alde suspectum est in sylva, hyems, stylus..." ("Y ist der griechische Buchstabe, der, genauso wie die anderen griechischen Buchstaben $\theta, \kappa, \varphi, \chi, \zeta$, nur in jenen Wörtern anerkannt werden muss, die sich offensichtlich vom Griechischen herleiten, und nicht wegen einer Vermutung der unnützen Grammatiker. $\langle\ldots\rangle\langle Y\rangle$ ist sehr fragwürdig in sylva, hyems, stylus...", Nolte 1744, col. 195). Über Noltes Werk s.: Solopov 2008, 40-41. Der neueste „Antibarbarus“ von Johann Philipp Krebs behandelt die Schreibweise von silva nicht (Krebs 1905-1907; s. über ihn: Solopov 2008, 75-78). Im etymologischen Wörterbuch von Ernout und 
Die höchst einflussreichen Lexikographen Johann Matthias Gesner und (in etwas verkürzter Form) Egidio Forcellini äußerten ähnliche Meinungen (Gesner 1749, T.4, 691 s. v. sylva [sic]; Forcellini 1771, T. 4, 134 s. v. silva).

Die bis ins 18. Jh. bestehende Notwendigkeit, gegen die Schreibung sylva zu kämpfen, erklärt auch die Schwankungen in der Schreibweise des abgeleiteten Vogelnamens. In der Überlieferung der Aristoteles-Übersetzung von Gaza wird er mit „““ geschrieben: silvia. ${ }^{52}$ Diese Schreibweise ist in Gessners „Pandectae“ wiederholt (Gessner 1548, f. 227r), allerdings wohl nur deswegen, weil es ein direktes Zitat aus Gazas Übersetzung ist. Für das Wort 'Wald' zog Gessner nämlich die Schreibung sylva vor: er hat als Äquivalent von ט̋ $\lambda \eta$ in seinem griechisch-lateinischen Wörterbuch eben das mit "y" geschriebenes Wort sylva angegeben (Gessner 1537, col. 1780; vgl. Gessner 1562, f. LII 4r). ${ }^{53}$ Dadurch lässt sich die Tatsache erklären, dass sich der Vogelname in Gessners „Historia animalium“ eben sylvia schrieb (Gessner 1555, 697)..$^{54}$ Die Autoren weiterer zoologischer Werke folgten fast ausnahmslos ${ }^{55}$ seinem Beispiel, als sie das Wort sylvia zunächst als eine alternative Bezeichnung für das Rotkehlchen und im 18. Jh. als neuen Tribus-, Gattungs- und Artnamen benutzten.

Obwohl Cuvier 1800 versuchte, die Schreibung silvia wiedereinzuführen, hat sich doch in der späteren Nomenklatur die 1769 von Scopoli verwendete Variante sylvia etabliert (vgl. Sherborne 2006).

\section{Zusammenfassung}

Es erwies sich also, dass die in der heutigen zoologischen Nomenklatur gültige Bezeichnung der Grasmücken, Sylvia, auf folgende Weise entstanden ist.

In einem Überlieferungszweig von Arist. Hist. an. existiert eine - immer noch unklar, ob richtige oder aus einem Kopistenfehler geborene - Lesart ü $\lambda a \varsigma$ (592b22). Um dieses Wort auf Latein wiederzugeben, hat Theodor Gaza in den 1470er Jahren die Lehnübersetzung silvia eingeführt. Da die entsprechende Stelle des griechischen Textes seit der 1504 erschienenen princeps in den Editionen immer anders gelesen wurde ( $\pi v \rho \rho o v ́ \lambda a \varsigma$ ), blieb die Herkunft des Wortes silvia bis jetzt unklar. Während der frühen Neuzeit änderte sich nicht nur die Schreibung des Vokals in der Wurzel, sondern auch die Bedeutung dieses Wortes. Im 16. Jh. wurde es willkürlich mit dem aristotelischen

Meillet wird über den Grund der Schreibweise sylva kurz bemerkt: „La graphie sylva est due au rapprochement de ü $\lambda \eta$ “ („Die Schreibung sylva ist einer Angleichung an ü $\lambda \eta$ geschuldet“, Ernout, Meillet 2001, 626).

${ }^{52}$ Ich habe sowohl die Handschriften aus dem Vatikan und Montecassino (s. oben Anm. 21) geprüft, als auch einige gedruckte Auflagen (Gaza 1476, f. 1 8v; 1504, f. 40v; 1524, f. 74r; 1668, 945), sowie den Tiernamensindex aus der griechisch-französischen Ausgabe von 1783 (Camus 1783, t. II, 11).

53 Über Gessners Wörterbuch s.: Sergeev 2014; Sergeev 2018, 71-77.

54 Im oben behandelten Vogelbuch von William Turner aus dem Jahr 1544 wird das Wort auch als sylvia geschrieben, aber Turners Usus konnte die spätere Tradition kaum beeinflussen, weil er sylvia nur flüchtig im Anhang erwähnt (vgl. oben Anm. 32). In Edward Wottons Traktat „De differentiis animalium“ wird das Wort als silvia geschrieben; dort wird es allerdings auch nur flüchtig erwähnt, neben erithacus und rubecula (Wotton 1552, f. 127v). Wottons Werk, zwar an sich höchst innovativ, hatte auf die Nomenklatur wesentlich weniger Wirkung als Gessners kurz darauf erschienene „Historia animalium“ (es ist bemerkenswert, dass Wotton der einzige ist, der einen seltsamen Druckfehler in Gazas Übersetzung erkannte, den vor und nach (!) ihm niemand bemerkt hat, vgl. Vorobyev 2018, 340).

${ }^{55}$ Beispielsweise wurde zwar die Form silvia in Gabryel Rzączyńskis „Historia naturalis curiosa regni Poloniae“ benutzt (Rzączyński 1721, 279), aber in der erweiterten Auflage durch sylvia ersetzt (Rzączyński 1745, 418), vgl. oben Anm. 40. 
Vogelnamen épiӨaкоৎ / rubecula gleichgesetzt, der für die Bezeichnung des Rotkehlchens gehalten wurde. Silvia wurde also zu einem raren Alternativnamen des Rotkehlchens. Im 18. Jh. wurde das Wort Sylvia in den Klassifikationen von Jacob Theodor Klein und Giovanni Antonio Scopoli als Name zwei verschiedener Vogeltaxa verschiedenen Umfangs verwendet. Dieser Name wurde sowohl von Klein, als auch von Scopoli deswegen benutzt, weil die beiden Taxa u. a. die Art Rotkehlchen enthielten. Schließlich wurde in der 1800 veröffentlichten Tierreichsklassifikation von Georges Cuvier der Umfang der Gattung Sylvia reduziert, sodass sie nur die Grasmücken enthielt, während die Rotkehlchen nun zu einer anderen Gattung Erithacus gehörten. Seit 1800 gilt also das Wort Sylvia als Name der Gattung „Grasmücken“.

\section{Literatur}

Adelung J. C. Grammatisch-kritisches Wörterbuch der Hochdeutschen Mundart. Teil 1. Leipzig, J. G. I. Breitkopf und Compagnie, ${ }^{2} 1793$ (Online-Version: http://www.zeno.org/Adelung-1793, letzter Zugriff am 7.8.2018).

Albin E. A natural history of birds. [Vol. 1]. London, printed for the author, sold by William Innys, John Clarke and John Brindley, 1731.

Aldrovandi U. Ornithologia. T.2. Bologna, apud Iohannem Baptistam Bellagambam, 1600.

Aristoteles Latinus Database (http://clt.brepolis.net/ald/Default.aspx, letzter Zugriff am 7.8.2018).

Aubert H., Wimmer F. (Hgg., Übers.). Aristoteles Thierkunde. Bd. 1-2. Leipzig, Wilhelm Engelmann, 1868.

Bairlein F., Bonan A. Old world warblers and parrotbills (Sylviidae), in: J. del Hoyo, A. Elliott, J.Sargatal, D. A. Christie, E. de Juana (Hgg.), Handbook of the birds of the world alive. Barcelona, Lynx Edicions, 2018 (https://www.hbw.com/node/52320, letzter Zugriff am 7.8.2018).

Balme D. M.(Hg.). Aristotle. Historia animalium. Vol.1: Books I-X: Text. Prepared for publication by A. Gotthelf. Cambridge, Cambridge University Press, 2002.

Bäumer Ä. Geschichte der Biologie. Bd. 2. Zoologie der Renaissance - Renaissance der Zoologie. Frankfurt a. M. u. a., Peter Lang, 1991.

Bäumer Ä. Geschichte der Biologie. Bd. 3. 17. und 18. Jahrhundert. Frankfurt a. M. u. a., Peter Lang, 1996.

Beekes R. S. P. Etymological dictionary of Greek. Vols. 1-2. Leiden - Boston, Brill, 2010.

Bekker I. (Hg.). Aristoteles Graece, edidit Academia Regia Borussica. Vol. 1. Berlin, Georg Reimer, 1831.

Berger F. Die Textgeschichte der Historia Animalium des Aristoteles. Wiesbaden, Ludwig Reichert, 2005.

Beullens P. Aristotle, his translators, and the formation of ichthyologic nomenclature, in: M. Goyens, P. De Leemans, A. Smets (Hgg.), Science translated. Latin and vernacular translations of scientific treatises in Medieval Europe. Leuven University Press, 2008, 105-122.

Beullens P., Bossier F. (Hgg.). Aristoteles. De historia animalium. Translatio Guillelmi de Morbeka. Pars 1: libri I-V. Leiden - Boston - Köln, Brepols, 2000.

Beullens P., Gotthelf A. Theodore Gaza's translation of Aristotle's De animalibus: content, influence and date. GRBS 2007, 47, 469-513.

Bezzel E. Kompendium der Vögel Mitteleuropas. Passeres. Singvögel. Wiesbaden, Aula Verlag, 1993.

Boehme R. L., Flint V.E. Pyatiiazychnyi slovar' nazvanii zhivotnykh. Ptitsy, Latinskii-russkii-angliiskii-nemetskii-frantsuzskii [Dictionary of animal names in five languages. Birds. Latin, Russian, English, German, French] (auf Russisch). Moskau, Russkii iazyk — Russo, 1994.

Bonitz H. Index Aristotelicus. Berlin, Georg Reimer, 1870.

Brisson M.-J. Ornithologie, ou méthode contenant la division des oiseaux en ordres, sections, genres, espèces et leurs variétés. T. 3. Paris, Jean-Baptiste Bauche, 1760.

[Bussemaker U.C.] (Hg.). Aristotelis opera omnia Graece et Latine. Vol.3. Paris, Ambroise Firmin Didot, 1854.

Camus A.-G.(Hg., Komm.). [Histoire des animaux d'Aristote]. T. 1. Histoire des animaux d'Aristote avec la traduction française. T. 2. Notes sur l'histoire des animaux d'Aristote. Paris, chez la veuve Desaint, 1783.

Chantraine P. Dictionnaire étymologique de la langue grecque. Histoire des mots. Paris, Klincksieck, ${ }^{2} 1999$.

Charleton W. Onomasticon zoicon. Mantissa anatomica. De variis fossilium generibus. London, apud Iacobum Allestry, 1668. 
Collar N. (2018). European Robin (Erithacus rubecula), in: J. del Hoyo, A. Elliott, J.Sargatal, D. A.Christie, E. de Juana (Hgg.), Handbook of the birds of the world alive. Barcelona, Lynx Edicions, 2018 (https:// www.hbw.com/node/58467, letzter Zugriff am 7.8.2018).

Collar N., Newton I., Bonan A. Finches (Fringillidae), in: J. del Hoyo, A. Elliott, J.Sargatal, D. A. Christie, E. de Juana (Hgg.), Handbook of the birds of the world alive. Barcelona, Lynx Edicions, 2018 (https:// www.hbw.com/node/52376, letzter Zugriff am 7.8.2018).

Comenius J. A. Orbis sensualium pictus. Die sichtbare Welt. Nürnberg, typis et sumptibus Michaelis Endteri, 1658.

Cuvier G. Tableau élémentaire de l'histoire naturelle des animaux. Paris, Baudouin, VI [1798].

Cuvier G. Leçons d’anatomie comparée. T. 1. Paris, Baudouin, VIII [1800].

Dittmeyer L. Untersuchungen über einige Handschriften und lateinische Übersetzungen der Aristotelischen Tiergeschichte. Programm des Königlichen Neuen Gymnasiums zu Würzburg für das Studienjahr 1901/02. Würzburg, Königliche Universitätsdruckerei von H. Stürtz, 1902.

Dittmeyer L. (Hg.). Aristotelis De animalibus historia. Leipzig, Teubner, 1907.

Dübner F., Lehrs F. S. (Hgg.) Manuelis Phile versus iambici de proprietate animalium, in: Poetae bucolici et didactici. Paris, Didot, 1862. Teil [3], mit eigener Pagination.

Erasmus D. Adagiorum chiliades tres, ac centuriae fere totidem. Venedig, in aedibus Aldi, 1508.

Ernout A., Meillet A. Dictionnaire étymologique de la langue latine. Histoire des mots. Paris, Klincksieck, ${ }^{4} 2001$.

Filius L.S.(Hg.). The Arabic version of Aristotle's Historia animalium. Book I-X of Kitāb al-Hayawān. Leiden - Boston, Brill, 2018 (im Druck).

Forcellini E., Facciolati J. Totius Latinitatis lexicon. T. 1-4. Padua, typis Seminarii, 1771.

Friedrich U. Naturgeschichte zwischen artes liberales und frühneuzeitlicher Wissenschaft. Conrad Gessners Historia animalium und ihre volkssprachliche Rezeption. Tübingen, Niemeyer, 1995.

Frisk H. Griechisches etymologisches Wörterbuch. Bd. 1-3. Heidelberg, Carl Winter, Universitätsverlag, 1960-1972.

Gaza T. (Übers.). Aristoteles. De animalibus. Venedig, Johann von Köln und Johann Manthen, 1476 (GW 2350).

Gaza T. (Übers.). Aristoteles, Theophrastus. De natura animalium. De partibus animalium. De generatione animalium. De historia plantarum. De causis plantarum. Venedig, Aldus Manutius, 1504.

Gaza T. et al. (Übers.). Aristoteles. Opera quae extant omnia. T. 3. Rom, typis Angeli Bernabò, 1668.

Gaza T., Alcionio P. (Übers.). De historia animalium. De partibus animalium et earum causis. De generatione animalium. De communi animalium gressu. De communi animalium motu. Paris, ex officina Simonis Colinaei, 1524.

Gesner J. M. Novus linguae et eruditionis Romanae thesaurus. T.1-4. Leipzig, impensis Caspari Fritschii viduae et Bernhardi Christiani Breitkopfii, 1749 (Online-Version: https://www2.uni-mannheim.de/ mateo/camenaref/gesner.html, letzter Zugriff 7.8.2018).

[Gessner C.] Lexicon Graecolatinum. Basel, in officina Ioannis Walderi, 1537.

Gessner C. Pandectarum sive partitionum universalium libri XXI, Zürich, excudebat Cristophorus Froschoverus, 1548.

Gessner C. Historiae animalium liber III qui est de avium natura. Zürich, apud Cristophorum Froschoverum, 1555.

Gessner C., Hartung J. $\Lambda$ EछIKON sive dictionarium Graecolatinum. Basel, ex officina Hieronymi Curionis, impensis Henrichi Petri, 1562.

Geus A. Klein, Jacob Theodor, in: Neue Deutsche Biographie, Bd. 11. Berlin, Duncker \& Humblot, 1977, 740-741 (Online-Version: https://www.deutsche-biographie.de/pnd117523216.html ndbcontent, letzter Zugriff am 7.8.2018).

Halleux R. (Hg.). Les alchimistes grecs. Vol. 1. Paris, Les belles lettres, 1981.

Helander H. On neologisms in Neo-Latin, in: P. Ford, J. Bloemendal, C. Fantazzi (Hgg.), Brill's encyclopaedia of the Neo-Latin world. 2 vols. Leiden - Boston, Brill, 2014, vol. 1, 37-54.

Hünemörder C. (Hg.). Aristoteles. De animalibus libri XIX in der Übersetzung des Michael Scotus. Nach der Handschrift Rom, Bibliotheca Apostolica Vaticana, Chigi E VIII 251 (s. XIII), fol. 1ra-108rb, mit Textkorrekturen aus den Handschriften Nürnberg, Stadtbibliothek, Cent. VI 10 und Pisa, Bibliotheca di Santa Caterina, Cod. 11. Buch I-XIV. [Eichstätt - München], 1994.

Jobling J. A. The Helm dictionary of scientific bird names. From Aalge to Zusii. London, Christopher Helm, 2010. 
Jobling J. A. Sylvia, in: Idem, Key to Scientific Names in Ornithology, in: J. del Hoyo, A. Elliott, J. Sargatal, D. A. Christie, E. de Juana (Hgg.), Handbook of the birds of the world alive. Barcelona, Lynx Edicions, 2018a (https://www.hbw.com/dictionary/definition/sylvia, letzter Zugriff am 7.8.2018).

Jobling J. A. Sylvia, in: Idem, Key to Scientific Names in Ornithology, in: J. del Hoyo, A. Elliott, J. Sargatal, D. A. Christie, E. de Juana (Hgg.), Handbook of the birds of the world alive. Barcelona, Lynx Edicions, 2018b (https://www.hbw.com/dictionary/definition/sylvia-0, letzter Zugriff am 7.8.2018).

Jonston J. Historia naturalis de avibus. Frankfurt a. M., impensa Matthaei Meriani, 1650.

Junius H. Nomenclator, omnium rerum propria nomina variis linguis explicata indicans. Antwerpen, ex officina Christophori Plantini, 1567.

Junius H. Nomenclator, omnium rerum propria nomina septem diversis linguis explicata indicans. Frankfurt a. M., excudebat Egnolphus Emmelius sumptibus Ionae Rosae viduae, 1620.

Kircher A. Arca Noe. Amsterdam, apud Joannem Janssonium a Waesberge, 1675.

Klein J. T. Historiae avium prodromus. Lübeck, apud Ionam Schmidt, 1750.

Krebs J. P. Antibarbarus der lateinischen Sprache. Bd. 1-2. Basel, Benno Schwabe, ${ }^{7} 1905-1907$.

Kretschmer P. Mythische Namen. 15. Lityerses und Hylas. Glotta 1925, 14 (1-2), 33-36.

Kristeller P. O. Iter Italicum. A finding list of uncatalogued or incompletely catalogued humanistic manuscripts of the Renaissance in Italian and other libraries. Vol. 1: Agrigento to Novara. London - Leiden, The Warburg Institute — Brill, 1977.

Krumbacher K. Geschichte der byzantinischen Litteratur von Justinian bis zum Ende des Oströmischen Reiches (527-1453). München, ${ }^{2} 1897$.

Liddell H. G., Scott R., Jones H. S. A Greek-English lexicon. Oxford, Clarendon Press, ${ }^{9} 1940$ (Online-Version: http://perseus.uchicago.edu/Reference/LSJ.html Source, letzter Zugriff am 7.8.2018).

von Linné C. Fauna Suecica. Leiden, apud Conradum Wishoff et Georgium Jacobum Wishoff, 1746.

von Linné C. Systema naturae. T. 1. Stockholm, impensis directis Laurentii Salvii, ${ }^{10} 1758$.

Louis P. (Hg., Übers.). Aristote. Histoire des animaux. T. 3. Livres 8-10. Paris, Les belles lettres, 1969.

Manutius A. (Hg.). Aristoteles. Opera. T.3. Venedig, in domo Aldi, 1497 (GW 2334).

Martens J., Päckert M. Kinglets and Firecrests (Regulidae), in: J. del Hoyo, A. Elliott, J. Sargatal, D. A. Christie, E. de Juana (Hgg.), Handbook of the birds of the world alive. Barcelona, Lynx Edicions, 2018 (https:// www.hbw.com/node/52307, letzter Zugriff am 7.8.2018).

Miglio M. (Hg.). Giovanni Andrea Bussi. Prefazioni alle edizioni di Sweynheym e Pannartz prototipografi romani. Mailand, Il Polifilo, 1978.

Monfasani J. Collectanea Trapezuntiana. Texts, documents, and bibliographies of George of Trebizond. Binghamton, Center for Medieval and Early Renaissance Studies, 1984.

Monfasani J. The Pseudo-Aristotelian Problemata and Aristotle's De Animalibus in the Renaissance, in: A. Grafton, N.Siraisi (Hgg.), Natural particulars. Nature and the disciplines in Renaissance Europe. Cambridge [Mass.] — London, The MIT press, 1999, 205-247.

Monfasani J. Aristotle as scribe of nature: the title-page of ms Vat. Lat. 2094. JWI 2006, 69, 193-205.

de Nebrija E. A. Dictionarium [Latinohispanicum et Hispanicolatinum]. Saragossa, s. n., 1513.

de Nebrija E. A. Dictionarium. Antwerpen, in aedibus Iohannis Steelsii, 1545.

Nolte J. F. Lexicon Latinae linguae antibarbarum quadripartitum. Leipzig - Helmstadt, apud Christianum Fridericum Weygand, 1744 (Online-Version: https://www2.uni-mannheim.de/mateo/camenaref/nolte.html, letzter Zugriff am 7.8.2018).

Orvieto P. (Hg.). Angelo Poliziano, Sylva in scabiem. Rom, Salerno editrice, 1989.

Pape W. Handwörterbuch der Griechischen Sprache. 4 Bdd. Bd. 2. Griechisch-Deutsches Wörterbuch. $\Lambda-\Omega$. Braunschweig, Friedrich Vieweg und Sohn, ${ }^{3} 1880$.

Perfetti S. 'Cultius atque integrius'. Teodoro Gaza, traduttore umanistico del De partibus animalium. Rinascimento 1995, 35, 253-286.

Perosa A. (Hg.) Angeli Politiani Sylva in scabiem. Rom, Edizioni di storia e letteratura, 1954.

Perotti N. Cornucopiae. Venedig, Paganinus de Paganinis, 1489 (GW M31093).

Ramminger J. Neo-Latin: character and development, in: P. Ford, J. Bloemendal, C. Fantazzi (Hgg.), Brill's encyclopaedia of the Neo-Latin world. 2 vols. Leiden - Boston, Brill, 2014, vol. 1, 21-36.

Riedl-Dorn C. Wissenschaft und Fabelwesen. Ein kritischer Versuch über Conrad Gessner und Ulisse Aldrovandi. Wien — Köln, Böhlau, 1989.

Rzączyński G. Historia naturalis curiosa regni Poloniae. Sandomir, typis Collegii Societatis Jesu, 1721.

Rzączyński G. Auctuarium historiae naturalis curiosae regni Poloniae, Magni Ducatus Lithuaniae annexarumque provinciarum. Danzig, s. n., 1745. 
Sabba F. La Bibliotheca universalis di Conrad Gesner. Monumento della cultura europea. Roma, Bulzoni editore, 2012.

Scaliger J. C. (Hg., Übers., Komm.) Aristotelis Historia de animalibus. Toulouse, typis Raymundi Colomerii, 1619.

Schneider J. G. (Hg., Komm.). Aristoteles. De animalibus historiae libri X. Graece et latine. T. I-IV. Leipzig, in bibliopolio Hahniano, 1811.

Scopoli G. A. Annus I historico-naturalis. Descriptiones avium... Leipzig, sumtibus Christophori Gottlob Hilscheri, 1769.

Sergeev M.L. Conrad Gessner (1516-1565) kak bibliograf [Conrad Gessner (1516-1565) as a bibliographer] (auf Russisch). Acta linguistica Petropolitana 2008, IV (1), 527-537.

Sergeev M.L. Grechesko-latinskii slovar' 1537 g.: svidetel'stva sostavitelia i izdatelia [The 1537 Greek-Latin dictionary: compiler's and editor's witness] (auf Russisch). Vox Medii Aevi 2014, 1, 40-46.

Sergeev M.L. 2018. Sopostavlenie iazykov v XVI veke (na primere "Mitridata" (1555) Conrada Gessnera) [Comparing languages in the sixteenth century (the case of Conrad Gessner's 1555 Mithridates)] (auf Russisch). Dissertation zur Erlangung des Grades des kandidat nauk. Sankt Petersburg, Russische Akademie der Wissenschaften, 2018.

Serra F. Synonymorum apparatus. Venedig, apud Baba, 1654.

Sherborne C.D. Index animalium. 2006 (Online-Database: http://www.sil.si.edu/DigitalCollections/indexanimalium/about/, letzter Zugriff am 7.8.2018). Ursprüngliche Ausgabe: Sherborn C.D. Index animalium sive index nominum quae ab A. D. MDCCLVIII generibus et speciebus animalium imposita sunt. Sectio prima. A Kalendis Ianuariis, MDCCLVIII usque ad finem Decembris, MDCCC. Cambridge, Cambridge University Press, 1902; Idem. Index animalium sive index nominum quae ab A. D. MDCCLVIII generibus et speciebus animalium imposita sunt. Sectio secunda. A Kalendis Ianuariis, MDCCCI usque ad finem Decembris, MDCCCL. London, Longsmans, Green \& Co., British Museum (Natural History), 1922-1932.

Sicherl M. Griechische Erstausgaben des Aldus Manutius. Druckvorlagen, Stellenwert, kultureller Hintergrund. Paderborn u. a.: Ferdinand Schöningh, 1997.

Solopov A. I. Nachala latinskoi stilistiki [Initia eloquentiae Latinae] (auf Russisch). Moskau, Indrik, 2008.

Springer K. B., Kinzelbach R. K. Das Vogelbuch von Conrad Gessner (1516-1565). Ein Archiv für avifaunistische Daten. Berlin - Heidelberg, Springer, 2009.

Statius. Sylvarum lib. 5, Thebaidos lib. 12, Achilleidos lib. 2. Warrington, veneunt apud G. Eyres typographum, 1778.

Stresemann E. Ornithology. From Aristotle to the present. Cambridge [Mass.] — London, Harvard University Press, 1975.

Sundevall C. J. Die Thierarten des Aristoteles von den Klassen der Säugethiere, Vögel, Reptilien und Insekten. Übersetzung aus dem Schwedischen. Stockholm, Samson \& Wallin, 1863.

Thompson D. W. A glossary of Greek birds. Oxford, Clarendon Press, 1895.

Turner W. Avium praecipuarum, quarum apud Plinium et Aristotelem mentio est, brevis et succincta historia. Köln, excudebat Ioannes Gymnicus, 1544.

Tyler S. White Wagtail (Motacilla alba), in: J. del Hoyo, A. Elliott, J. Sargatal, D. A.Christie, E. de Juana (Hgg.), Handbook of the birds of the world alive. Barcelona, Lynx Edicions, 2018 (https://www.hbw. com/node/57821, letzter Zugriff am 7.8.2018).

Veenhusen J. (Hg.) Publii Papinii Statii Sylvarum lib. 5, Thebaidos lib. 12, Achilleidos lib. 2. Leiden, ex officina Hackiana, 1671.

Vorobyev G. M. Neologizmy Feodora Gazy iz ego latinskogo perevoda „De animalibus“ Aristotel'a v sovremennoi zoologicheskoi nomenklature [Theodore Gaza's neologisms in his translation of Aristotle's De animalibus and their reception in modern zoology] (auf Russisch). Indoevropeiskoe iazykoznanie i klassicheskaia filologiia 2015, 19, 158-168.

Vorobyev G. M. O prirode latinskogo nazvaniia ryby sargiacus (Arist. Hist. an. 610b6) [On the Latin fish name sargiacus (Arist. Hist. an. 610b6)] (auf Russisch). Indoevropeiskoe iazykoznanie i klassicheskaia filologiia 2018, 22 (1), 333-345.

Willoughby F. Ornithologiae libri tres. London, impensis Joannis Martyn, 1676.

Wotton E. De differentiis animalium. Paris, apud Vascosanum, 1552. 
Sylvia: о происхождении научного названия славки (Arist. Hist. an. 592b22)

\section{Григорий Михайлович Воробъев}

Институт лингвистических исследований РАН, Российская Федерация, 199053, Санкт-Петербург, Тучков пер., 9;

Институт новолатинских исследований им. Людвига Больцмана, Лангер вег 11, Инсбрук, 6020, Австрия; g_voro01@uni-muenster.de

В статье доказывается, что слово sylvia, используемое в качестве научного названия

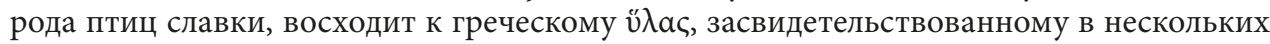
рукописях «Истории животных» Аристотеля (592b22). В третьей четверти XV в. при

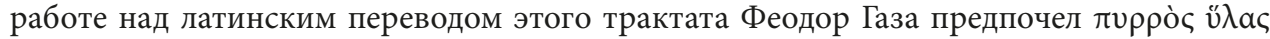

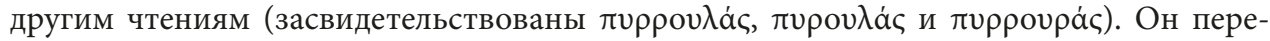

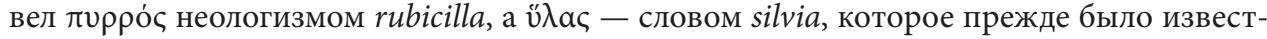
но только в качестве имени собственного. Поскольку все издания греческого текста, начиная c editio princeps 1504 г., принимают предложенное Альдом Мануцием чтение

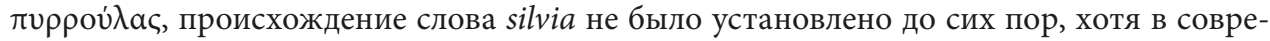
менных изданиях, снабженных критическим аппаратом, приводится и альтернатив-

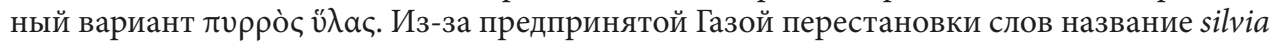
ошибочно сочли синонимом другого введенного им неологизма, rubecula (перевод со-

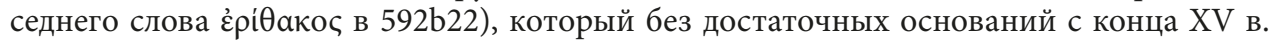
отождествляли с зарянкой. Поэтому в 1769 г. Джованни Антонио Скополи использовал слово sylvia (с XVI в. за ним закрепилось написание через "у») в качестве обозначения введенного им нового рода птиц, включавшего, среди прочего, зарянок. В 1800 г. Жорж Кювье сузил объем этого рода: он оставил в нем только славок, а другие виды, в том числе зарянок, выделил в несколько новых родов. Итак, слово silvia, введенное Феодором Газой для перевода неизвестного, возможно, появившегося в результате

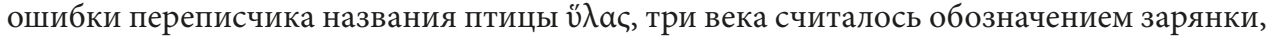
a c 1800 г., в форме sylvia, обозначает род славки.

Ключевые слова: зоологическая номенклатура раннего Нового времени, латинские неологизмы, История животных Аристотеля, славки, зарянки, sylvia, silvia, pyrrhula, erithacus, rubecula. 